\title{
Tachyon dynamics of the black disc limit in the hard small $x$ processes in QCD.
}

\author{
B. Blok \\ Department of Physics, Technion-Israel Institute of Technology, 32000 Haifa, Israel \\ L. Frankfurt \\ School of Physics and Astronomy, Raymond and Beverly Sackler Faculty of Exact Sciences, \\ Tel Aviv University, 69978 Tel Aviv, Israel

\begin{abstract}
We investigate the effective field theory (EFT) which gives the approximate description of the scattering of the two hard small dipoles in the small $x$ processes in QCD near the black disc limit(BDL). We argue that the perturbative QCD approaches predict the existence of tachyon and visualize it in the approximation where $\alpha_{P}^{\prime}=0$. We demonstrate that the high energy behavior of the cross-section depends strongly on the diffusion law in the impact parameter plane. On the other hand, almost threshold behavior of the cross section of the hard processes and multiplicities, i.e. fast increase of cross sections (color inflation), melting of ladders into color network and softening of the longitudinal distributions of hadrons are qualitatively insensitive to the value of diffusion in the impact parameter space. We evaluate $\alpha_{P}^{\prime}$ near the black disk limit and find significant $\alpha_{P}^{\prime}$ as the consequence of the probability conservation.
\end{abstract}




\section{INTRODUCTION}

One of the distinctive features of the hadron collisions at high energies is the increase of the cross sections with the energy which is often parameterized as the intercept of the Pomeron $\alpha_{P}(t=0)>1$, cf. review in ref. [1]. It follows from the unitarity of the $S$ matrix and the analytic properties of amplitudes in the momentum transfer plane that the cross sections of hadron-hadron collisions can not exceed the limit $\sigma \leq c_{h} \ln ^{2}\left(s / s_{o}\right)$ [2, 3], with the coefficient $c_{h}$ determined by the radius of the pion cloud of a hadron [4]. At achievable energies the contribution of the Pomeron exchange instead of the projectile scattering of the pion cloud dominates in the peripheral collisions because pion is a pseudo-goldstone meson of spontaneously broken chiral symmetry [5], cf. analysis of the role of pion cloud in high energy processes in ref. [6]. The account of this property of QCD explains why the observed value of $c_{h}$ is significantly smaller than that arising from the black scattering off the pion periphery of a hadron [5]. Moreover, in the case of the interaction increasing with energy the complete absorption for the central hadron-hadron collisions at ultra-high energies arises as the consequence of the numerically large and increasing with the energy number of constituents in the wave functions of the colliding hadrons. At ultrahigh energies the coefficient $c_{h}$ should be the same for all hadrons and nuclei [5].

The modelling of the hadron collisions at high energies within the eikonal approximation where single Pomeron exchange is used as "potential" indicated that the unitarity limit should be achieved at collider energies since the intercept of the Pomeron is $\alpha_{P}(t=0) 1$, [7, 8, 9]. The analysis of data on elastic pp collisions found that the scattering at central impact parameters achieves unitarity limit at energies of FNAL [9, 10]. At the domain of energies to be achieved at LHC blackness of pp interaction will occupy significantly wider region in the impact parameter space.

It seems now that the discussed above pattern of behavior of amplitudes of high energy processes is similar to that expected at sufficiently small $x$ in perturbative QCD for the cross sections of hard processes. One of the striking properties of the hard processes in the perturbative QCD is the rapid increase of interaction with energy [11, 12, 13, 14]. Such behavior can not continue for arbitrary small $x$, otherwise it will violate the probability conservation. It was understood recently that this property of perturbative QCD and increase with energy of the number of constituents within a projectile lead to complete absorption for the central colorless dipole -hadron collisions. cf. [17]. Thus at ultra-high energies the total cross sections of deep-inelastic scattering (DIS) should achieve the unitarity limit 15] $: \sigma($ dipole + Nucleon $) \rightarrow c_{D} \log ^{3}\left(x_{0} / x\right)$ with the universal coefficient at ultra-high energies. (Additional $\log x_{0} / x$ as compared to the hadronic cross sections is due to the ultraviolet divergence of the electromagnetic charge, cf. also ref.[16]). Both theoretical calculations, of the elastic scattering of colorless gluon dipole and the analysis of the data on hard diffraction in DIS observed at HERA, indicate that the black disc regime (BDL) seems to be achieved for gluon distributions (but not for quark distributions) at $Q^{2} \sim$ few $\mathrm{GeV}^{2}$ within the proton at the verge of the kinematics of HERA. The blackness of interaction including the hard one will be important in the central pp collisions in the significantly wider region in the kinematics of LHC, where it will compete with the hadronic final states in the new particles production, for the review and references see ref. [17].

In the previous paper [18] we deduced within the WKB approximation Lagrangian of effective field theory (EFT) describing the interactions of quasiparticles-pQCD color neutral ladders relevant for small $x$ behavior of single scale hard processes in the vicinity of BDL. This 
Lagrangian accounts for the perturbative QCD calculations of small $x$ behavior of amplitudes as an input. We found that the perturbative QCD dynamics of the single scale hard processes at small $x$ predicts the presence of tachyon type behavior of amplitudes of physical processes in EFT. In particular, the increase with distance of the matrix elements of the correlators between currents evaluated within the perturbative QCD indicates instability of produced pQCD system which is an important feature of the onset of the critical phenomena [19]. (Note however that part of the increase of correlator with distance follows from the Lorentz slow down of interaction. This phenomenon resembles turbulence [19]). The existence of the tachyon leads to the spontaneously broken continuous symmetries and related variety of critical phenomena.

At the intermediate stage of our analysis we were able to use similarity between the EFT and the model for high energy hadron collisions based on preQCD Reggeon Calculus with the intercept $\alpha_{P}(t=0)>1$ thorougly analyzed in cf. refs. [20, 21, 22, 23, 24]. Reformulating to hard processes in QCD near the black disk regime the results, obtained within this model, we found kinks, spontaneous violation of translational invariance and related two dimensional "phonons". At the same time the finding that within the EFT the pQCD ladders are overlapped in space and time rather often necessitates to go beyond the approximations leading to EFT and to account for the the interchange by constituents between overlapping ladders . Account of exchange by color leads to color network - effect beyond EFT. Thus a variety of new physical phenomena which are absent in refs. [20, 21, 22, 23, 24] have been found in ref. [18]: melting of pQCD ladders and formation of color network, importance of tunneling transitions $\propto \exp \left(-1 / \alpha_{s}\right)$.

In the approach developed in ref. [18] the important role is played by the "Pomeron" slope $\alpha_{P}^{\prime}$ that has not been calculated in $\mathrm{pQCD}$ so far. Indeed, the slow convergence of the pQCD series at small $x$, cf. refs. 14, 25], precludes the reliable pQCD calculation of the value of $\alpha_{P}^{\prime}$. Besides, the significant value of $\alpha_{P}^{\prime}$ arises near BDL because of the fast increase with energy of pQCD ladder. For the estimates of $\alpha_{P}^{\prime}$ near the black disk regime see discussion below and ref. [17.

To visualize the similarities between small $x$ physics in QCD and critical phenomena and the importance of V.Gribov diffusion in small $x$ regime we consider in this paper the alternative approximation $\alpha_{P}^{\prime}=0$ and compare the obtained results with those obtained in ref. [18]. The evident advantage of the approximation $\alpha_{P}^{\prime}=0$ is the possibility of the analytical solution that made it possible to visualize the origin of the new QCD phenomena found in ref. [18]. Note that because of a dynamical generation of $\alpha_{P}^{\prime} \neq 0$ near the BDL, in the physical situation one can not put $\alpha_{P}^{\prime}=0$ in eq. 1.1. However the analysis that equates $\alpha_{P}^{\prime}$ to zero will give a useful information on the dependence of different properties of the theory on the theoretical input.

A number of alternative approaches to the ultra small $\mathrm{x}$ behavior of hard processes has been suggested and developed in the literature, first of all the color condensate approach 26, 27, 28, 29, 30] and related unitarization of the perturbative QCD contributions within the the eikonal approximations, see i.e. refs. [31, 32]. In difference with these approaches we accounted for in ref. 18] ladder loops, found physical vacuum within the WKB approximation and accounted for the tunneling transitions between the physical and the perturbative QCD vacua. This lead to the existence of the variety of new QCD phenomena cf. ref. 18] such as melting of ladders and formation of color network, spontaneous violation of two dimensional translation symmetry and existence of two dimensional "phonons", color inflation. Note that the black disk limit behavior does not follow from the color glass con- 
densate approach, at least if multiladder loops are neglected [33]. In addition, let us note that the color glass condensate approach as well as the other leading order BFKL related approaqches, are characterized by diffusion to small distances in the transverse plane at high energies, i.e. the color condensate is contained in narrow threads. On the other hand, in EFT the diffusion to large distances dominates, and the high energy densities may arise due to the rapid increase of the number of constituents.

Technically the effective field theory of ref. [18] has been derived for one-scale processes such as the scattering of two equally small size dipoles, where the evolution of scale is a correction that we will neglect.

This theory is governed by the Hamiltonian [18]

$$
\begin{aligned}
L & =1 / 2\left(p \partial_{y} q-q \partial_{y} p\right)-\alpha^{\prime} p \triangle_{b} q-\mu p q-\kappa p q(p+q) \\
& -c_{\text {dipole }} \int \exp (-b Q / 2) q(y, \vec{B}-\vec{b}) d^{2} b \delta(y+Y) \\
& -c_{\text {dipole }} \int \exp (-b Q / 2) p(y, \vec{B}-\vec{b}) d^{2} b \delta(y-Y),
\end{aligned}
$$

Here $p=i \psi^{+}(y, \vec{b}), q=i \psi(y, \vec{b})$ are operators of production and annihilation of the pQCD ladders , $y=\log \left(x_{o} / x\right)$ is rapidity, $\vec{b}$ is the impact parameter, $\mu$ is a "Pomeron" intercept, and $\kappa$ is a three-ladder vertex. We took $\kappa$ to be constant near the BDL which properly accounts for hard QCD physics. On the contrary within the LO BFKL approximation $\kappa$ is singular as a function of the momentum transfer $t$ [34]. Such a bizarre behavior demonstrates that physics of large transverse distances artificially suppressed within LO BFKL approximation gives actually significant contribution within this approximation. This is because within LO BFKL approximation there is no separation of scales between soft and hard QCD phenomena , no running coupling constant and no Sudakov form factors. The value $c_{\text {dipole }}$ is determined by the normalisation of the dipole wave function.

In ref. [35] the slope $\alpha_{P}^{\prime}$ has been put to zero from the beginning. The latest NLO calculations [14] show that the rapid diffusion to large nonperturbative distances characteristic for the leading order BFKL approximation is suppressed when the next to leading order approximation is accounted for. However the randomness of the gluon radiation and the related Gribov diffusion in impact parameter space should be there [36]. We will find significant value for $\alpha_{P}^{\prime}$ in the kinematics near the black disk limit, see discussion below.

To visualize the sensitivity of QCD phenomena to the value of $\alpha_{P}^{\prime}$ it is interesting to investigate what will happen if to put it to zero. This will show quantatively, how the asymptotic behavior of the cross-section is influenced by the diffusion in the transverse plane. As we shall see shortly, the diffusion in a transverse plane plays a crucial role. For example, the account of V.Gribov diffusion in the impact parameter space leads to Froissart behavior [18], while as we shall see below the absence of diffusion leads within EFT to grey disc-asymptotic constant value of the cross-sections. Physical cross section still increases with energy because of effects neglected in EFT.

The paper is organized in the following way. In section 2 we formally consider the case $\alpha_{P}^{\prime}=0$ to visualize presence of tachyon, to study the qualitative effect of V.Gribov diffusion in impact parameter space on the high energy behavior of structure functions. In section 3 
we argue that physical value of $\alpha_{P}^{\prime}$ is significant and can not be neglected at high energies. We summarize our results in the conclusion.

\section{EFFECTIVE FIELD THEORY FOR SMALL SIZE DIPOLE SCATTERING WITHOUT DIFFUSION.}

The Lagrangian of the EFT without V.Gribov diffusion in impact parameter space is given by eq. 1.1, with $\alpha_{P}^{\prime}=0$. Thus the Lagrangian is

$$
\begin{aligned}
L & =1 / 2\left(p \partial_{y} q-q \partial_{y} p\right)-\mu p q-\kappa p q(p+q) \\
& -c_{\text {dipole }} \delta(y) q f \\
& -c_{\text {dipole }} p \delta(y-Y)
\end{aligned}
$$

Recall that the first term accounts for the kinetic energy, the second corresponds to "Pomeron" intercept $1+\mu$. The vertex $\kappa$ is effectively a 3 -reggeon vertex.

One approach to the theory is to solve the classical equations of motion that follow from the Lagrangian 2.1 and then consider the quasiclassical fluctuations around this solution.

The equations of motion for the Hamiltonian corresponding to eq. 2.1 can be easily solved. Indeed, these equations of motion can be written as

$$
\frac{d p}{d y}=-\frac{d H}{d q} \quad \frac{d q}{d y}=\frac{d H}{d p}
$$

where

$$
H=-\mu p q+\kappa p q(p+q)
$$

Since the energy is a constant of motion, we can put $H=E$, obtain $p=p(q, E)$, and then explicitly solve the second of equations 2.2 for q. There are four critical points for this action:

$$
(p, q)=(0,0),(0, \mu / \kappa),(\kappa / \mu, 0)
$$

with $E=0$, and

$$
(p, q)=(-\mu /(3 \kappa),-\mu /(3 \kappa))
$$

with $E<0$. The S-matrix in the classical approximation is given by

$$
S \sim \exp \left(-A_{\mathrm{cl}}\right)
$$

where we need to calculate the classical action $A_{\mathrm{cl}}$ for each value of $\mathrm{E}$, then take the minimal one, and consider fluctuations around it. It turns out that the dominant contribution arises from the trajectories with $E \rightarrow 0$, that corresponds to the trajectories connecting the perturbative and nonperturbative vacua with $\mathrm{E}=0$. (we refer the reader to the ref. [37] for the detailed analysis of the trajectories). These trajectories are the quantum mechanical analogues of the kinks of the $2+1$ dimensional theory [18]. Evidently this approach can be easily extended to the arbitrary number of dimensions cf. citeBF. 
The approximation 2.1 is a nonhermitean quantum mechanics. It can be reformulated by substituting variables into Hermitian quantum mechanics. After the transformation: $q=x^{2}, p=d / d\left(x^{2}\right)$, and transformation of the Hamiltonian

$$
H=\exp \left(-F(x) H_{0} \exp (F(x),\right.
$$

where

$$
F(x)=(1 / 4)\left(x^{2}-\mu / \kappa^{2}\right)^{2},
$$

we obtain the Hermitean Hamiltonian cf. [24, 38]

$$
H=-\kappa / 4 \frac{d^{2}}{d x^{2}}+V(x)
$$

where

$$
V(x)=(\kappa / 4)\left(x^{2}\left(x^{2}-\mu / \kappa\right)^{2}+\mu / \kappa-x^{2}\right)
$$

This Hamiltonian determines the same Borel convergent series for $\mu<0$ as the original one . So both theories are equivalent, at least for the low energy levels we are interested for. The derived interaction in the Hermitian quantum mechanics is the double-well (Higgstype) potential. In the case $\mu>0$ predicted by the perturbative QCD the theory undergoes the transition similar to a Higgs phenomena: there are two degenerate vacua (see Fig 1), while the perturbative vacuum corresponds to the maximum of $\mathrm{V}$ and a tachyon. Double well structure of the interaction clearly visualizes the origin of the tachyon predicted by the pQCD calculations, the similarity between the BDL in hard QCD and second order phase transitions. The spontaneous violation of the continuous symmetries, in the case when $\alpha_{P}^{\prime}$ is kept nonzero, makes the analogy with second order phase transitions even more close.

The S-matrix is determined by

$$
S(f, Y)=<0\left|\exp \left(-c_{\text {dipole }} P\right) \exp \left(-H_{0} Y\right) \exp \left(-c_{\text {dipole }} q\right)\right| 0>
$$

in terms of the original nonhermitian hamiltonian and can be expressed in terms of the eigenfunctions and eigenvalues of the equivalent hermitean hamiltonian [24, 38]:

$$
S=\frac{\sum_{n}<\phi_{0}\left|\exp (-F) O_{2} \exp (F)\right| \phi_{n}><\phi_{n}\left|O_{1}\right| \phi_{0}>\exp -\left(E_{n}-E_{0}\right) Y}{<\phi_{0}\left|\exp (F) O_{2} \exp (F)\right| \phi_{n}><\phi_{n}\left|O_{1}\right| \phi_{0}>},
$$

where $\mathrm{F}(\mathrm{x})$ is given by the equation 2.6 .

$$
\begin{gathered}
O_{1}=\exp \left(-c x^{2}\right), \\
O_{2}=\exp \left(+c \frac{\delta}{\delta_{x^{2}}}\right),
\end{gathered}
$$

and $<\phi_{0}|O| \phi_{n}>$ means $\int_{-\infty}^{\infty} \phi_{0}(x) O \phi_{n}(x) d x$.

We shall briefly formulate here the main results which are the same for both approaches discussed above:

A) The cross section of hard processes in high energy limit is constant. The value of the constant depends on the relation between the source strength and the ratio $\mu / \kappa$.

We consider in the paper weak coupling limit for single scale hard processes $\alpha_{s} N_{c}<<1$ where, the cross-section tends to

$$
\sigma_{Y \rightarrow \infty} \exp \left(-c_{\text {dipole }} \mu / \kappa\right) \sim \text { const }
$$


In other words, one gets instead of Froissart limit $\left(\sigma(s) \sim \log ^{2}(s)\right.$ behavior) a grey disk behavior.

B) Moreover, the tunnel transitions in this case lead (albeight at extremely high $y \sim$ $\exp \left(\mu^{2} / \kappa^{2}\right)$ energies) to slow decrease of the cross-section. Indeed, the S-matrix is controlled by the lowest energy level in this double-well. This level is aroused due to nonperturbative effects and is $E_{1} \sim \exp \left(-\mu^{2} /\left(2 \kappa^{2}\right)\right)$, thus the cross-section has a plato for

$$
\exp \left(\mu^{2} /\left(2 \kappa^{2}\right)\right)>>y>>1 / \mu .
$$

. The cross-section in this plato is given by eq. 2.11 For larger rapidities

$$
\sigma \sim 1 / s^{\exp \left(-\mu^{2} /\left(2 \kappa^{2}\right)\right)}
$$

The approach to the grey disk limit corresponds to "color inflation" phenomena, argued in ref. [18]. Indeed [24] the cross-section is well approximated by:

$$
\sigma \sim c_{\text {dipole }}^{2} \exp (\mu Y) /\left(1+\exp \left(c_{\text {dipole }} \mu / \kappa\right)\right) \exp (\mu Y)
$$

In other words the cross-section shows a step like behaviour with a step width of order $1 / \mu$ in rapidity space (Fig. 2).

Note that grey disc limit has academic interest only since in realistic case $\alpha_{P}^{\prime}$ can not be neglected near BDL because of renormalization due to tachyon effects,see the discussion below.

In the approximation where $\alpha_{D}^{\prime}=0$ all ladders are located at the same impact parameters. So overlap of ladders found in [18] should be even more valid in this case. Thus melting of overlapping ladders due to exchange by constituents and formation of color network are important even in the case when diffusion in impact parameter space is neglected.

\section{THE EVALUATION OF $\alpha_{P}^{\prime}$ NEAR THE BLACK DISK LIMIT.}

In the kinematics near the black disk limit where the partial waves become close to 1 the large value of $\alpha_{P}^{\prime}$ becomes important because of the rapid increase with energy of pQCD amplitude. This restriction due to the probability conservation has not been considered in the formulation of EFT in ref. [18] where $\mu, \alpha^{\prime}$ were parameters of a bare "Pomeron". The requirement of the self-consistency of the approximation put strong restriction on the value of the renormalized $\alpha_{P}^{\prime}$. The general expression for partial wave is $\operatorname{Im} f=c s^{\mu} \exp (-b Q)$. Here $b$ is the impact parameter, $Q$ is the scale of the discussed processes. For the sake of estimate we assumed that for the peripheral scattering the pQCD ladder should dominate. Since $\operatorname{Im} f \leq 1$ the important impact parameters can be evaluated from the condition $\operatorname{Im} f=1$ which is achieved within the EFT-cf. [18]. At $x=Q^{2} / s \rightarrow 0$ this gives: $b_{\max } Q=\ln \left(s / Q^{2}\right)$. Here $b_{\max }$ is the maximal impact parameter $\mathrm{b}$ for which $\operatorname{Imf}=1$. Thus $\alpha_{P}^{\prime}$ defined as the derivative of the slope of $\mathrm{t}$ dependence of the square of the amplitude is given by the formulae: $\alpha_{P}^{\prime} \equiv 1 / 2\left(d / d \ln \left(s / s_{o}\right)\right) b_{\max }^{2}$ cf. [17]. Thus near the black disk limit the renormalized $\alpha_{P}^{\prime}$ for hard processes should be even larger than that for soft QCD processes. So the limit $\alpha_{P}^{\prime}=0$ is unphysical, but it helps to visualize different aspects of QCD physics relevant for the black disk regime. 


\section{CONCLUSION.}

We demonstrate that the asymptotic behavior of the high energy processes strongly depends on the diffusion in the impact parameter space. On the other hand, the sharp increase of the multiplicities and of the cross section the during transition from the perturbative to the asymptotic regime, melting of pQCD ladders and formation of color network, and the softening of the forward distributions over the longitudinal momenta seem to be a diffusionindependent phenomena.

We argue that in a physical case of the hard energy scattering in QCD near the black disk limit the $\alpha_{P}^{\prime}$ value can not be small, because of the renormalization of the parameters of the effective Lagrangian due to the tachyon effects (the phenomenon neglected in refs. [18, 20, 21, 22, 23, 24, 37, 38]. In other words the diffusion in impact parameter space can never be neglected for physical problems, although it is legitimate to investigate it's influence on the cross-sections in the approximate approaches . 
[1] S.Donnachie, G. Dosch, P.Landshof and O.Nachtmann, Pomeron physics and QCD, Cambridge University Press, 2002.

[2] W. Hmeisenberg, Z. Phys. 133 (1952) 65.

[3] M. Froissart, Phys. Rev. 123 (1961) 1053.

[4] A. Martin, Phys. Rev., 129 (1963) 1432.

[5] L. Frankfurt, M.Strikman, M. Zhalov, Phys.Lett., B537 (2004) 51.

[6] D. Kharzeev,Invited talk at 39th Rencontres de Moriond on QCD and High-Energy Hadronic Interactions, La Thuile, Italy, 28 Mar - 4 Apr 2004, hep-ph/0408091

[7] K.A. Ter-Martirosyan, JET Lett., 11 (1970) 267, Yad. Fiz. 10 (1970) 1047, Nucl.Phys.B36 (1972) 566-574.

[8] A. Kaidalov, Phys. Repts, 50 (1979) 157.

[9] M. Blok, F. Halsen, Phys.Rev.D72:036006,2005, Erratum-ibid.D72:039902,2005.

[10] M.M. Islam, R.J. Luddy, A.V. Prokudin, Mod.Phys.Lett.,A18 (2003) 743.

[11] D.Gross and F.Wilczek, 1,Phys. Rev. D8 (1973) 3633; Phys. Rev. D9 (1974) 980.

[12] Yu.L. Dokshitser, Sov. Phys. JETP 46 (1977)

[13] E. Kuraev, V. Fadin, L. Lipatov, Sov. Phys.-JEP, 44 (1976) 443; 45 (1977) 199. I. Balitsky and L. Lipatov, Sov. J. Nucl. Phys., 28 (1978) 822.

[14] M. Ciafaloni, D. Colferai,G.P. Salam and A. Stasto, Phys. Lett. B541 (2002) 314, M. Ciafaloni, D. Colferai, G.P. Salam, Phys. Rev. D60 (1999) 114036.

[15] V. Guzey, L. Frankfurt, M. Strikman,M. McDermott, Phys.Rev.Lett., 87 (2001) 192301.

[16] V. Gribov, Sov. Phys., JETP 30 (1970) 709.

[17] L. Frankfurt, M. Strikman and C. Weiss, Ann. Rev.of Particle and Nucl.Science 2005, hep-ph/0507286.

[18] B. Blok and L. Frankfurt, hep-ph/0508218

[19] B.Blok and L.Frankfurt,Phys.Rev.D70 (2004) 094003.

[20] D. Amati, M. Ciafaloni, Le Bellac, G. Marchesini, Nucl. Phys. B112 (1976) 107.

[21] D. Amati, M. Ciafaloni, G. Marchesini, G.Parisi, Nucl. Phys. B114 (1976) 483.

[22] V. Alessandrini, D. Amati, M. Ciafaloni, Nucl.Phys. B130 1977) 429.

[23] V. Alessandrini, D. Amati, R. Jengo, Nucl Phys, B108 (1976) 425.

[24] M. Ciafoloni and G. Marchesini, Nucl. Phys., B105 (1976) 113; B109 (1976) 261.

[25] V. Fadin and L. Lipatov, Phys. Lett., B429 (1998) 127.

[26] L. McLerran and R. Venugopalan, Physical Review, D49 (1994), 2233,3352; D50 (1994) 2225; D59 (1999) 094002; see also L. McLerran, Surveys High Energy Phys., 18 (2003) 101; Nucl. Phys. A702 (2002) 49, for the latest reviews on the subject.

[27] J. Jalalian-Marian, A. Kovner, L. McLerran, H. Weigert, Phys. Rev., D55, (1997) 5414; J. Jalalian-Marian,A. Kovner,H. Weigert, Phys. Rev. D59 (1999) 014015; J. Jalalian-Marian,A. Kovner, A. Leonidov,H. Weigert, Phys. Rev. D59 (1999) 014014; Nucl. Phys. B504, 415; A. Kovner, M. Giulherme Milano, H. Weigert, Phys. Rev. D62, (2000) 114005.

[28] A. Mueller and A.Shoshi, Small x physics near the saturation regime, hep-ph/0405205, submitted to 39 Recontres de Moriond in QCD.

[29] L.McLerran,Nucl.Phys., A752 (2005) 355;

[30] E. Iancu, hep-ph/0510264, hep-ph/0510265.

[31] A. H. Mueller, Nucl. Phys. B415 (1994) 373; Yu. Kovchegov, Phys. Rev. D60 (1999) 034008; 
I. Balitsky, Nucl.Phys., B463 (1996) 99.

[32] E.Gotsman,E. M. Levin,U.Maor, Phys. Rev. D49 (1994) 4321; Phys. Lett. B347 (1995) 424.

[33] A. Kovner and U. Wiedemann, Phys.Lett., B551 (2003) 311; Phys. Rev. D66 (2002) 034031.

[34] J. Bartels, M. G. Ryskin and G. P. Vacca, Eur. Phys. J. C 27 (2003) 101.

[35] L. Lipatov, Phys. Repts., 286 (1997) 131.

[36] V. N. Gribov, 'Space-Time Description Of Hadron Interactions At High Energies, hep-ph/0006158.

[37] D. Amati, L.Caneshi and R. Jengo, Nucl. Phys., B101 (1975) 397.

[38] D. Amati, V. Alessandrini and R.Jengo, Nucl.Phys. B108 (1976) 425; R.Jengo, Nucl.Phys. B108 (1976) 447; M. Ciafaloni and E. Onofri, Nucl. Phys., B151 (1979 ) 118; M. Ciafaloni, M.Le Bellac and G.C. Rossi, Nucl. Phys. B130 (1977) 388; M. Ciafaloni, Nucl. Phys. B146 (1978) 427. 


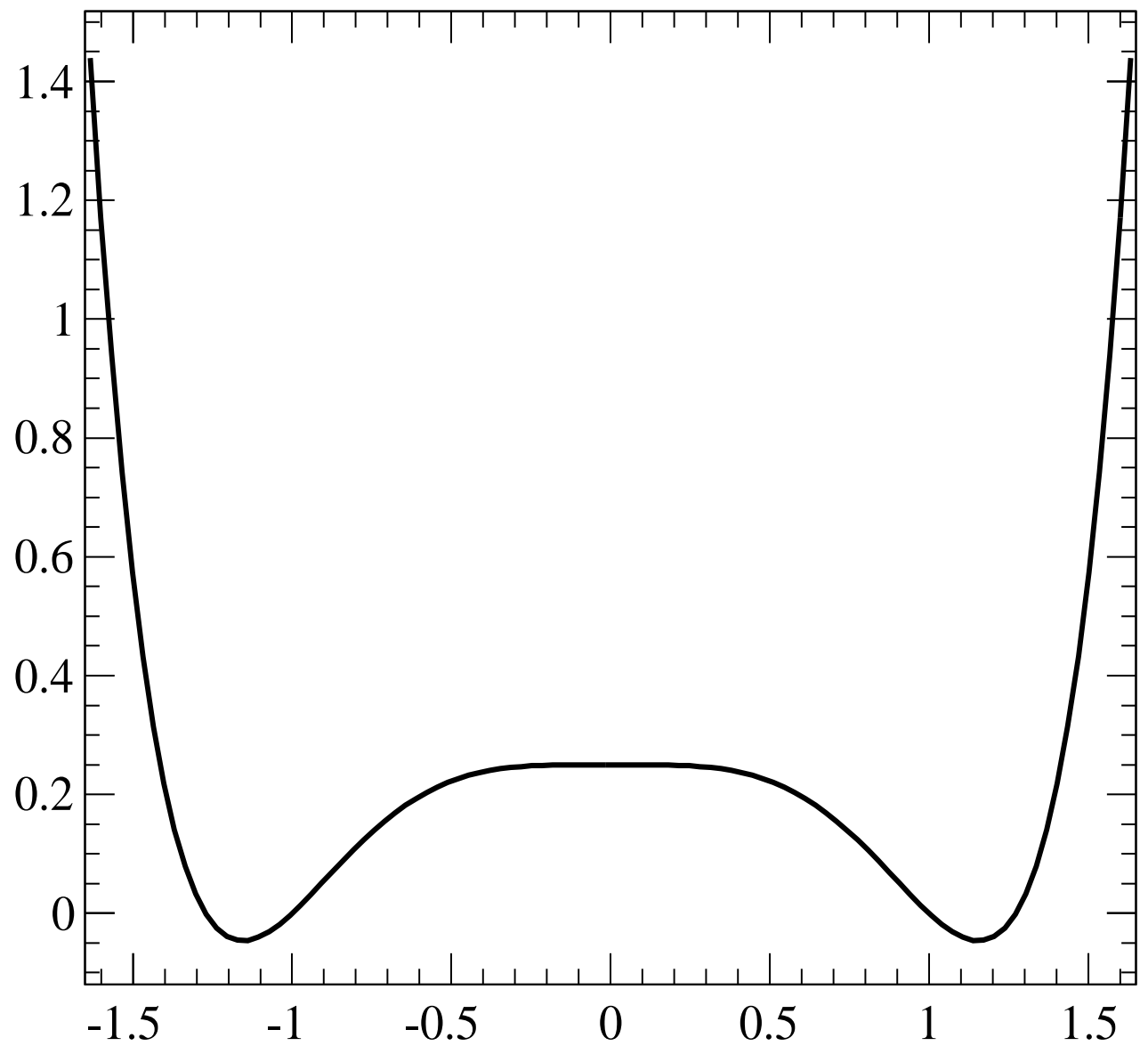

FIG. 1: The effective potential for $\mu>0$ (tachyon is present) 


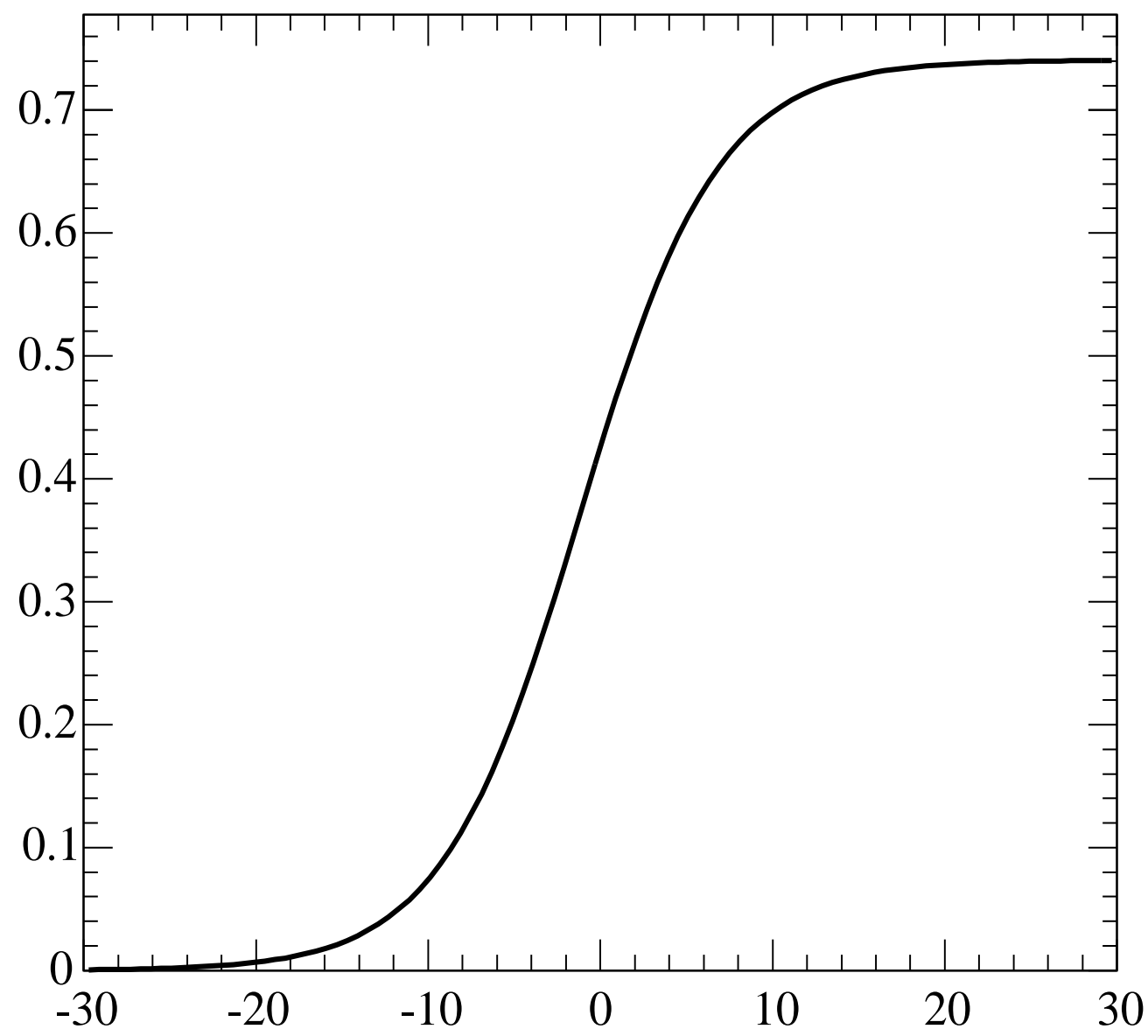

FIG. 2: The cross-section dependence on rapidity Y (color inflation) 